\title{
Synthesis of Thinned Concentric Circular Antenna Arrays Using Modified TLBO Algorithm
}

\author{
Zailei Luo, ${ }^{1}$ Xueming He, ${ }^{2}$ Xuedong Chen, ${ }^{1}$ Xin Luo, ${ }^{1}$ and Xiaoqing Li ${ }^{1}$ \\ ${ }^{1}$ State Key Laboratory of Digital Manufacturing Equipment and Technology, Huazhong University of Science and Technology, \\ Wuhan 430074, China \\ ${ }^{2}$ Public Security Marine Police Academy, Ningbo, Zhejiang 315801, China
}

Correspondence should be addressed to Xiaoqing Li; xqli@hust.edu.cn

Received 18 March 2015; Revised 28 June 2015; Accepted 1 July 2015

Academic Editor: Ahmad Safaai-Jazi

Copyright (C) 2015 Zailei Luo et al. This is an open access article distributed under the Creative Commons Attribution License, which permits unrestricted use, distribution, and reproduction in any medium, provided the original work is properly cited.

\begin{abstract}
Teaching-learning-based optimization (TLBO) algorithm is a new kind of stochastic metaheuristic algorithm which has been proven effective and powerful in many engineering optimization problems. This paper describes the application of a modified version of TLBO algorithm, MTLBO, for synthesis of thinned concentric circular antenna arrays (CCAAs). The MTLBO is adjusted for CCAA design according to the geometry arrangement of antenna elements. CCAAs with uniform interelement spacing fixed at half wavelength have been considered for thinning using MTLBO algorithm. For practical purpose, this paper demonstrated SLL reduction of thinned CCAAs in the whole regular and extended space other than the phi $=0^{\circ}$ plane alone. The uniformly and nonuniformly excited CCAAs have been discussed, respectively, during the simulation process. The proposed MTLBO is very easy to be implemented and requires fewer algorithm specified parameters, which is suitable for concentric circular antenna array synthesis. Numerical results clearly show the superiority of MTLBO algorithm in finding optimum solutions compared to particle swarm optimization algorithm and firefly algorithm.
\end{abstract}

\section{Introduction}

Concentric circular antenna array (CCAA) is a planar array with multiple concentric circular rings, which has many advantages over other array geometries. Firstly, the radiation pattern covers the whole space. The main lobe could scan in all directions and provide $360^{\circ}$ azimuth coverage without a significant change in beam pattern. Besides, compared with linear arrays and rectangular arrays, CCAAs are less sensitive to mutual coupling due to the lack of edge elements. For these reasons, CCAAs have been applied extensively to a variety of applications over more than 40 years, such as sonar, radar, and satellite communications systems [1-4].

Considering the design of CCAA, side lobe level (SLL) reduction is the key issue, since uniformly excited CCAA usually has high SLL. To make SLL lower, the number of antenna elements as well as the corresponding positions and excitations should be considered. Among those methods which can lower SLL, thinning technique is an important one. Thinning an array means turning OFF some selected elements in a uniformly spaced or periodic array to create a desired amplitude density across the aperture. The target of thinning is to reduce the number of antenna elements and SLL while maintaining nearly the same narrow beamwidth as for a filled array of equal size. Another attractive feature of thinning technique is the fact that the turned OFF elements can reduce the cost and complexity of antenna array. In general, the research on thinning antenna arrays dates back to 1960 s when statistical thinning methods were introduced to design low SLL arrays [5]. Most deterministic methods can handle only small dimensional problems such as linear arrays and small planar arrays [6-8]. The simulated annealing is a powerful stochastic searching method for problems with large dimensions, and it has been imposed to design thinned array for both narrow band and wide band arrays [9-11]. Recently, a variety of evolutionary algorithms have been utilized to design thinned antenna arrays [1214]. Basu and Mahanti optimized a CCAA with two rings using firefly algorithm (FA). Both uniformly excited CCAA and nonuniformly excited CCAA have been discussed in 
[15]. Singh and Kamal [16] present thinned CCAA design using biogeography-based optimisation (BBO) algorithm. Numerical results showed the BBO performed better results for design of uniformly excited CCAA with ten rings, as compared to modified particle swarm optimization (MPSO) [17] and differential evolutionary (DE) [18]. Singh and Rattan also proposed FA to handle the same problem effectively in their later work [19]. Binary FA and PSO were introduced for thinned concentric ring array antenna design in [20], which achieved the SLL reduction in all azimuth planes in steps of $1.8^{\circ}$.

In the previous works, most studies considered the CCAA with uniform excitation, which simplifies the optimization problem and only gets the local optimal solution. Further, in the abovementioned CCAA studies except [20], SLL reduction was only achieved in the phi $=0^{\circ}$ plane, which cannot be accepted for real application. Moreover, when it comes to the phased array which has many steering angles, an extended space needs to be considered to get satisfactory SLL. To this end, this paper will focus on two important issues of CCAA: nonuniform CCAA design for better array pattern and SLL reduction of CCAA in the whole regular and extended space.

TLBO is a recently developed algorithm which simulates the educational process of teaching and learning in the traditional classroom [21]. The main advantage of TLBO is less algorithm specified parameters; it requires only common controlling parameters like population size and number of generations. It has been regarded as a new rising star of evolutionary algorithms and successfully applied in many engineering optimization problems $[22,23]$. In our previous work, a modified version of teaching-learning-based optimization (MTLBO) was proposed for thinning and weighting rectangular antenna arrays [24]. In the present paper, the MTLBO is adjusted to design CCAAs, with which both uniformly and nonuniformly excited CCAAs are optimized for SLL reduction in the whole regular and extended space. Numerical results are given and compared with those from previous works to show the validity of the proposed method.

\section{Geometry, Array Factor, and Objective Function}

In concentric circular antenna array, all antenna elements are arranged in multiple concentric rings. Figure 1 shows the general configuration of CCAA with $M$ concentric circular rings, where the $m$ th $(m=1,2, \ldots, M)$ ring has a radius $r_{m}$ and the corresponding number of elements is $N_{m}$. Assuming that all the array elements are isotropic sources, then the beam pattern can be described by its array factor, which is given as follows [25]:

$$
\mathrm{AF}=\left|\sum_{m=1}^{M} \sum_{n=1}^{N} I_{m n} \exp \left[j k r_{m}\left(\cos \varphi_{m n} u+\sin \varphi_{m n} v\right)\right]\right|,
$$

where $k=2 \pi / \lambda$ is the wave number and $\varphi$ and $\theta$ are the azimuth and elevation angle, respectively. $r_{m}=N_{m} d_{m} / 2 \pi$ is the radius of the $m$ th ring, $d_{m}$ is the interelement spacing of the $m$ th ring, $I_{m n}$ is the excitation amplitude of the $n$th

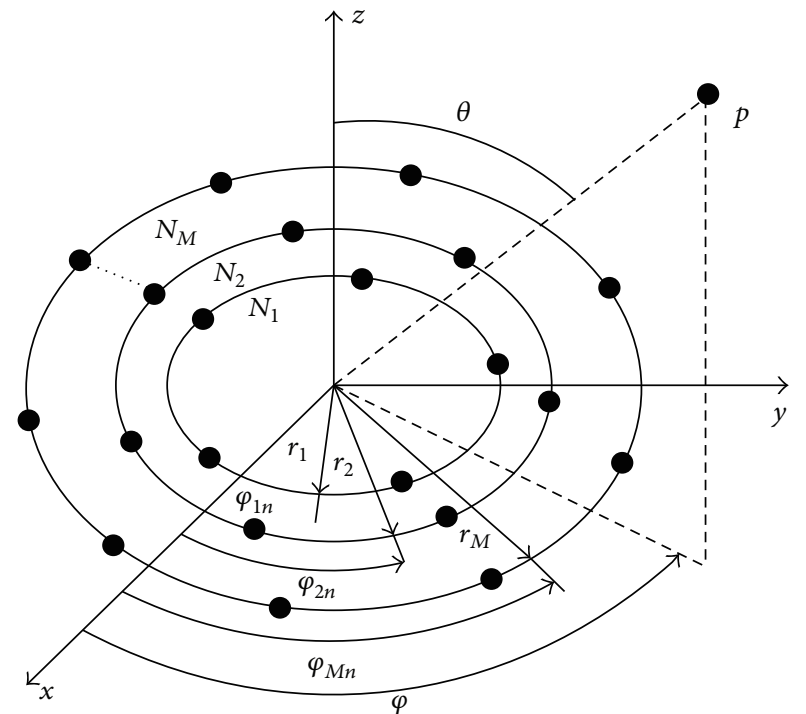

FIGURE 1: Geometry of concentric circular antenna array.

element of the $m$ th ring, and $\varphi_{m n}=2 \pi(n-1) / N_{m}$ is the angular position of the $n$th element of the $m$ th ring. The notation of $u, v$ indicates the unit direction:

$$
\begin{aligned}
& u=\sin \theta \sin \varphi-\sin \theta_{0} \sin \varphi_{0}, \\
& v=\sin \theta \cos \varphi-\sin \theta_{0} \cos \varphi_{0} .
\end{aligned}
$$

The pair $\left(\theta_{0}, \varphi_{0}\right)$ indicates the steering direction and the pair $(\theta, \varphi)$ indicates the arrival direction. In this paper, two kinds of $u-v$ space were considered for SLL reduction: one is regular $u-v$ space where the steering direction $\left(\theta_{0}, \varphi_{0}\right)=$ $(0,0)$, and $u, v \in[-1,1]$. The other is extended $u-v$ space where the steering direction $\left(\theta_{0}, \varphi_{0}\right) \neq(0,0)$. So the value of $u$ and $v$ varies according to the steering direction and belongs to $[-2,2]$ for any combination of the arrival and steering directions. The adoption of the $u-v$ space allows one to synthesize an array configuration that produces a beam pattern with desired profile for whatever steering direction.

In antenna array problems, there are many parameters that can be used to evaluate the objective (or cost) function such as gain, SLL, beamwidth, and size. Here, the goal is to design thinned arrays with minimum side lobes levels and at the same time keeping the beamwidth as small as possible. Thus, the following objective function is given as

$$
\begin{aligned}
F= & \mathrm{SLL}_{\max }+f\left(\mathrm{FNBW}_{o}-\mathrm{FNBW}_{d}\right) \\
& +f\left(\mathrm{Na}_{o}-\mathrm{Na}_{d}\right),
\end{aligned}
$$

where $\mathrm{SLL}_{\max }$ is the value of maximum SLL and FNBW and $\mathrm{FNBW}_{d}$ are the obtained and desired value of first null beamwidth, respectively. $\mathrm{Na}_{o}$ and $\mathrm{Na}_{d}$ are the obtained and desired value of number of turned ON elements. $f(x)$ is the constrained function defined as follows:

$$
f(x)= \begin{cases}x, & \text { if } x>0, \\ 0, & \text { if } x \leq 0 .\end{cases}
$$


In this work, antenna elements positions are kept fixed and the elements can have only two states, that is, "ON" $\left(I_{m n}>0\right)$ or "OFF" $\left(I_{m n}=0\right)$. An element in "ON" state contributes to the total array pattern. In contrast, an element is in "OFF" state if it is either passively terminated to a matched load or is open circuited, in which case it makes no contribution to the total array pattern. Thus, for the design of thinned CCAAs with minimum SLL, the optimization problem is to search for the excitation amplitudes $I_{m n}$ that are turned ON or OFF to accomplish this.

\section{Modified TLBO Algorithm}

3.1. The Original TLBO. Teaching-learning-based optimization (TLBO) is a recently proposed population based algorithm, which simulates the educational process of teachinglearning in the traditional classroom [18]. There are two basic components of the TLBO algorithm: teacher and learner, and the performance of the algorithm is summarized in two main phases: "teacher phase" and "learner phase." The "teacher phase" means that the learners learn from the teacher, which makes the algorithm proceed by shifting the mean of the learners towards their teacher. The "learner phase" means learners learn through the interaction among themselves, which increases diversity of knowledge of the whole class.

An analogy between the teaching-learning process and the optimum design of thinned concentric circular antenna arrays can be described in the following way: a class and a student in that class, respectively, represent the population and the candidate solution. Each subject taught to the learners represents a design variable (excitation amplitude of each element), and a class member's result is analogous to the value of objective function. The solution with best result in the entire population is defined as the teacher. The teachinglearning process is continued until reaching the termination criteria like other nature-inspired methods.

3.2. Motivation of the MTLBO Algorithm. According to the original TLBO algorithm, learners can improve their results by two basic modes of learning, through teacher (known as teacher phase) and through interaction with the other learners (known as learner phase), while the teacher gets no improvement from the class. It is well known that teacher is the highly learned person who shares knowledge with learners, and a good teacher gives learners better results. Hence the improvement of teacher's knowledge is a very important aspect of the whole class. To this end, we introduce an additional self-learning phase to original TLBO algorithm for the purpose of improving the result of teacher during the teaching-learning process. It should be noted that there are several papers that mentioned self-learning phase of TLBO algorithm $[26,27]$. In those papers, self-learning phase means that class members are self-motivated and improve their knowledge by self-learning. However, there are usually hundreds of elements that need to be optimized in CCAA optimization problem, and the self-learning among all class members will increase the computational costs greatly. Thus, it is more proper to introduce an extra self-learning phase to the teacher, which will improve the global search capability of TLBO while keeping the computational costs acceptable.

Synthesis of thinned CCAA means searching the optimum configuration of antenna array to obtain the desired array pattern. Specifically to the antenna elements of CCAA, it means choosing the proper state for each element. An element has two possible states, namely, ON or OFF, to change in the procedure of design thinned CCAA. To help the teacher improve his or her performance, a state transition search method (i.e., self-learning phase) is imposed in the MTLBO algorithm. During the state transition search method, all elements are randomly selected in a random sequence to test state transition. If the state of chosen element is OFF, it will be switched to $\mathrm{ON}$ and excited with a random amplitude; on the other hand, if the chosen element is $\mathrm{ON}$, it will be switched to OFF, namely, changing the excitation amplitude to 0 . The result of teacher determines whether such state transition will be accepted or not. A better result means the state transition is successful, and a worse result means the state transition is failing. It should be noted that a perturbation will be added to the turned $\mathrm{ON}$ element which failed in state transition to search for a better solution. The flow chart of state transition search method is expressed in Figure 2.

3.3. Procedures of MTLBO Algorithm. Implementation steps of the MTLBO algorithm are summarized as follows.

Step 1 (initialization). Initialize the population $X$ in a searching space bounded by a matrix with $P z$ (number of class members, i.e., class size) rows and $M N$ (number of antenna elements) columns. The objective function of the generation $g$ is given by

$$
\text { class }=\left[\begin{array}{cccc}
I_{1,1}^{g} & I_{1,2}^{g} & \cdots & I_{1, M N}^{g} \\
I_{2,1}^{g} & I_{2,2}^{g} & \cdots & I_{2, M N}^{g} \\
\vdots & \vdots & \vdots & \vdots \\
I_{P z, 1}^{g} & I_{P z, 2}^{g} & \cdots & I_{P z, M N}^{g}
\end{array}\right] \longrightarrow\left[\begin{array}{c}
F\left(X_{1}^{g}\right) \\
F\left(X_{2}^{g}\right) \\
\vdots \\
F\left(X_{P z}^{g}\right)
\end{array}\right] .
$$

Then select the class member who has the best knowledge (i.e., best solution, $F\left(X^{g}\right)_{\min }$ ) as the teacher $X_{t}^{g}$.

Step 2 (self-learning phase of teacher). In this phase, the teacher $X_{t}^{g}$ tries to improve his or her knowledge through self-learning with the state transition search method. Unlike rectangular antenna array, the antenna elements of CCAA are arranged according to the radius of rings. Antenna elements in different rings have different interelement spacing and contribute differently to the total array pattern. So the searching order of state transition search method needs to be adjusted for synthesizing CCAAs. The searching process starts from the inner ring to the outer ring, and elements on the same ring will be selected with equal probability. Once all the elements in the inner ring have been selected, the search will go to the next ring until all antenna elements of array have been selected.

Step 3 (teacher phase). During this phase, the teacher $X_{t}^{g}$ tries to increase the mean result of the class depending on his 


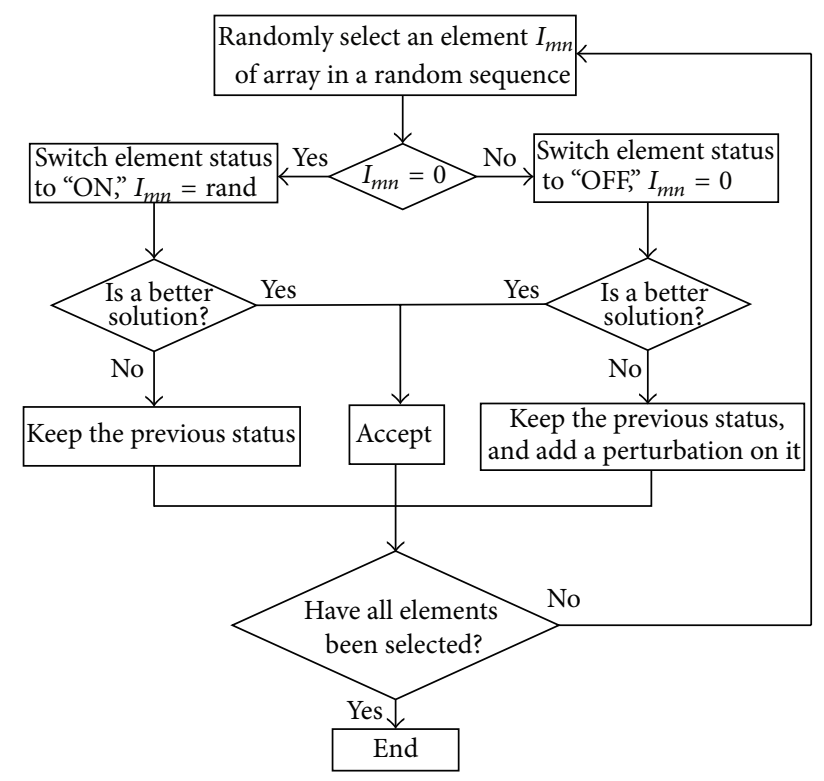

FIGURE 2: Flow chart of state transition search method.

or her knowledge. The solution is updated according to the difference between the existing mean result of learners and teacher, which is given by

$$
\text { Different_Mean }{ }^{g}=\operatorname{rand} \times\left(X_{t}^{g}-T_{F} \bar{X}^{g}\right) \text {, }
$$

where rand is the random number in the range $[0,1] ; X^{g}$ is the mean value of all the taught subjects in the whole class. $T_{F}$ is the teaching factor which determines the mean of value to be changed. For purpose of reducing algorithm specified parameter, the proposed MTLBO sets the same $T_{F}$ as the original TLBO. The value of $T_{F}$ is decided randomly with equal possibility as either 1 or 2 , which is corresponding to situation where learners learn nothing from the teacher or learn all the things from the teacher, respectively [28]. The existing solutions of learners will be updated according to the following expression:

$$
X_{\text {new }, j}^{g}=X_{\text {old }, j}^{g}+\text { Different_Mean }{ }^{g} .
$$

$X_{\text {new, } j}^{g}$ will be accepted and maintained if it gives a better value of objective function.

Step 4 (learner phase). Learners increase their knowledge by interaction among themselves. The leaner who has the worse value of objective learns from the other leaner according to the difference between them. The learning method of this phase is expressed as follows:

$$
\begin{aligned}
& \text { For } i=1: P z \\
& \text { Randomly select two learners } X_{i}^{g} \text { and } X_{j}^{g} \text {, where } i \neq j \\
& \text { If } F\left(X_{i}^{g}\right)<F\left(X_{j}^{g}\right) \\
& X_{\text {new, } j}^{g}=X_{j}^{g}+\operatorname{rand} \times\left(X_{i}^{g}-X_{j}^{g}\right) \\
& \text { Else }
\end{aligned}
$$

$$
\begin{aligned}
& X_{\text {new }, i}^{g}=X_{i}^{g}+\operatorname{rand} \times\left(X_{j}^{g}-X_{i}^{g}\right) \\
& \text { End if } \\
& \text { End for } \\
& \text { Accept } X_{\text {new }}^{g} \text { if it gives a better function value. }
\end{aligned}
$$

Step 5 (termination). If the predetermined maximum number of generations or the goal of optimization is achieved, the search will stop; otherwise go to Step 2.

\section{Numerical Results and Comparisons}

Numerical experiments for CCAA carried out in [20] were performed to verify that the proposed MTLBO algorithm is able to obtain better performance than other published methods. The target CCAA has a single element in center and seven surrounding rings; each ring of the antenna contains $8 m(m=1,2, \ldots, 7)$ elements. In the MTLBO implementation, the class size is set as 50 , and the number of generations is taken as 200. It should be noted that the variables of MTLBO are continuous, while the variables of objective function $I_{m n}$ are discrete in the uniform excited array. So the values of variables needed to be discredited to calculate the objective function in each generation. Specifically, $I_{m n}$ are rounded to one and zero in the uniformly excited CCAAs. As for the nonuniformly excited CCAAs, a threshold $\tau$ is introduced to determine whether the element is "ON" $\left(I_{m n}>\right.$ $\tau$ ) or "OFF" $\left(I_{m n} \leq \tau\right)$. In our case, the threshold $\tau$ is set as 0.2 ; namely, the current amplitude $I_{m n} \leq 0.2$ will be set to zero. All the tests were run with MATLAB on a PC equipped with an Intel Core G630 (2.7 GHz) provided with $2 \mathrm{~GB}$ of RAM memory.

4.1. SLL Reduction in the Regular Space. In this example, the target CCAA was thinned for SLL reduction in the regular $u-v$ space, where the steering angle $\left(\theta_{0}, \varphi_{0}\right)=(0,0)$ and the values of $u, v$ range from -1 to 1 , which is the same as in [20]. The binary firefly algorithm (BFA) and binary particle swarm optimization (BPSO) were imposed to synthesize the target CCAA. In the CCAA optimized by BFA, the $\mathrm{Na}$ is 148 , SLL is $-17.84 \mathrm{~dB}$, and the FNBW is 0.13 which is the same as that of full array. In the CCAA optimized by BPSO, the $\mathrm{Na}$ is 142 , SLL is $-16.98 \mathrm{~dB}$, and the FNBW is also 0.13. As for the proposed MTLBO, we can get the optimized CCAA with less active elements and better SLL, while keeping the FNBW the same as 0.13 . The configuration of uniform CCAA optimized by MTLBO algorithm and the corresponding array factor are shown in Figure 3.

Besides the uniform CCAA case, we considered the design of nonuniform CCAA with MTLBO algorithm. One of the optimized results is shown in Figure 4, the final $\mathrm{Na}$ is 130 , SLL is $-19.93 \mathrm{~dB}$, and the FNBW is 0.13 . Figure 5 shows the comparison of array factors in the phi $=0^{\circ}$ plane. Comparisons between the best results of the proposed MTLBO algorithm and that of others are given in Table 1. It is clearly shown that the proposed MTLBO obtained a better array factor with fewer turned ON elements than others' work. 


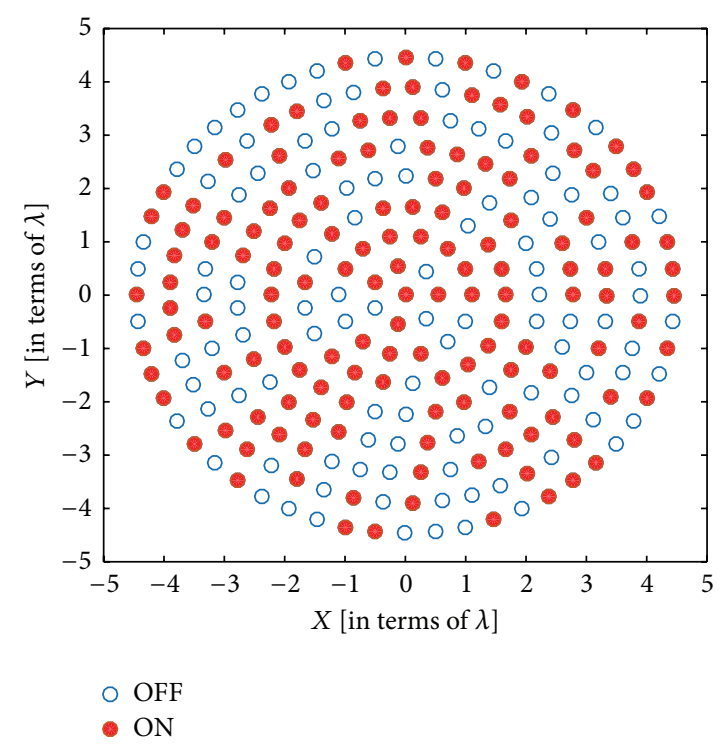

(a)

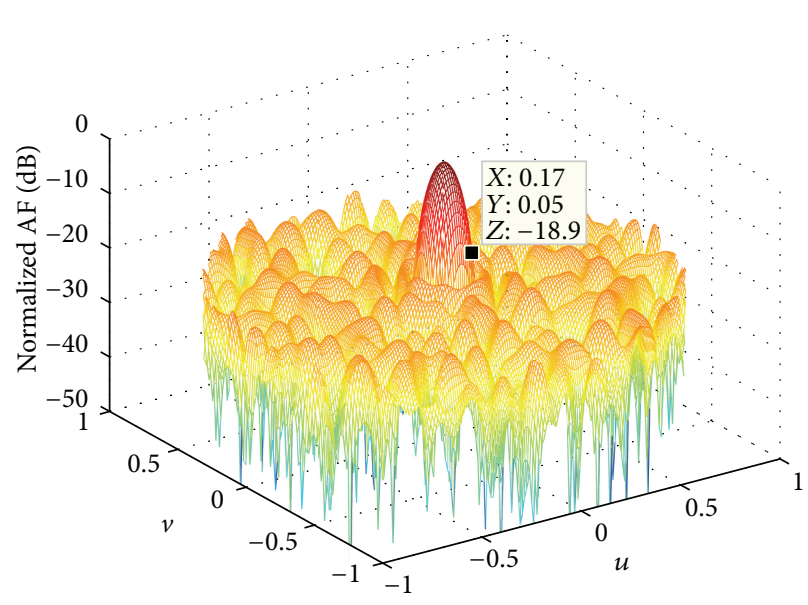

(b)

FIGURE 3: Uniform CCAA optimized by MTLBO for SLL reduction in general space. (a) Configuration of a 141-element uniform array and (b) the related array factor with $\mathrm{SLL}=-18.9 \mathrm{~dB}$ and $\mathrm{FNBW}=0.13$.

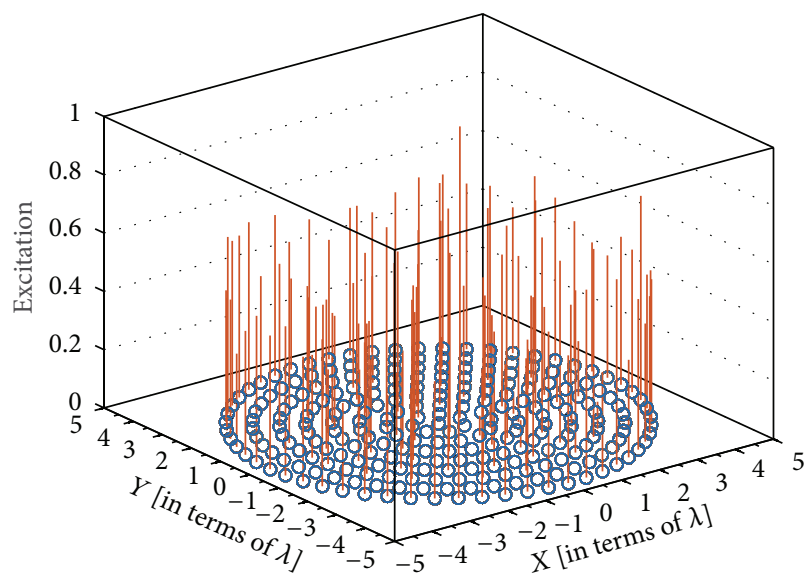

(a)

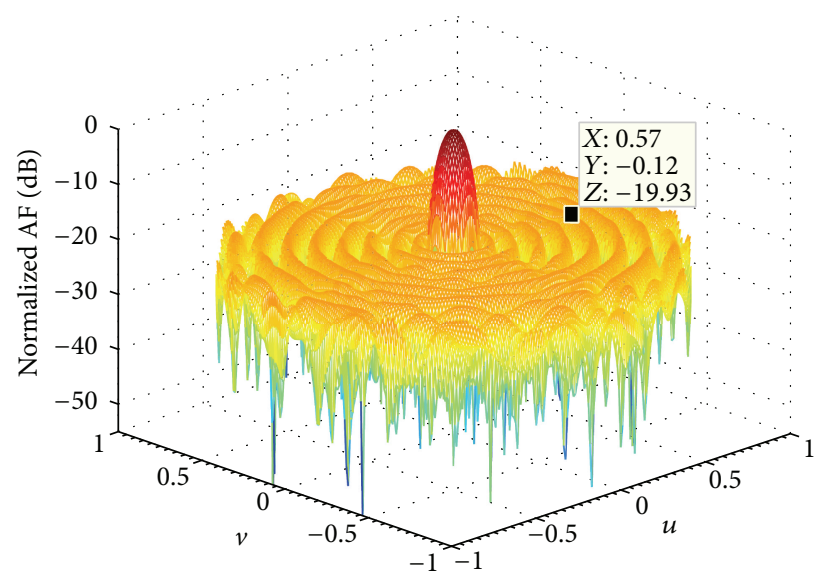

(b)

FIgURE 4: Nonuniform CCAA optimized by MTLBO for SLL reduction in regular space. (a) Configuration and excitation of a 130-element nonuniform array and (b) the related array factor with SLL $=-19.93 \mathrm{~dB}$ and FNBW $=0.13$.

TABLE 1: The SLL reduction in regular $u-v$ space performance comparison of different methods.

\begin{tabular}{lcccc}
\hline Excitation & Algorithms & $\mathrm{Na}$ & SLL (dB) & FNBW \\
\hline \multirow{4}{*}{ Uniform } & BFA in [20] & 148 & -17.84 & 0.13 \\
& BPSO in [20] & 142 & -16.98 & 0.13 \\
& Proposed MTLBO & 141 & -18.90 & 0.13 \\
\hline Nonuniform & Proposed MTLBO & 130 & -19.93 & 0.13 \\
\hline
\end{tabular}

4.2. SLL Reduction in the Extended Space. In this section, the objective of thinned CCAA design is to get SLL reduction in the extended $u-v$ space. Firstly, we considered a phased array system with the $u-v$ space extended to $[-1.5,1.5]$, which is adequate in most phased imaging sonar systems [11]. The uniform and nonuniform thinned CCAA were optimized by MTLBO with good SLL and FNBW performance. Figure 6 shows the optimized uniform CCAA with 156 ON elements, the corresponding array factor is presented by Figure 6(b) with a maximum SLL of $-19 \mathrm{~dB}$, and the FNBW is 0.13 . The nonuniform CCAA was also optimized by MTLBO, and the configuration and related array factor are given in Figure 7. In the optimized nonuniform CCAA, there are only $152 \mathrm{ON}$ elements to obtain a lower SLL with the same FNBW.

As for some extreme situation, where phased array requested for wide steering angles, the $u-v$ space needs a further extension. To this end, we considered the design of thinned CCAA for SLL reduction in the adequately extended 


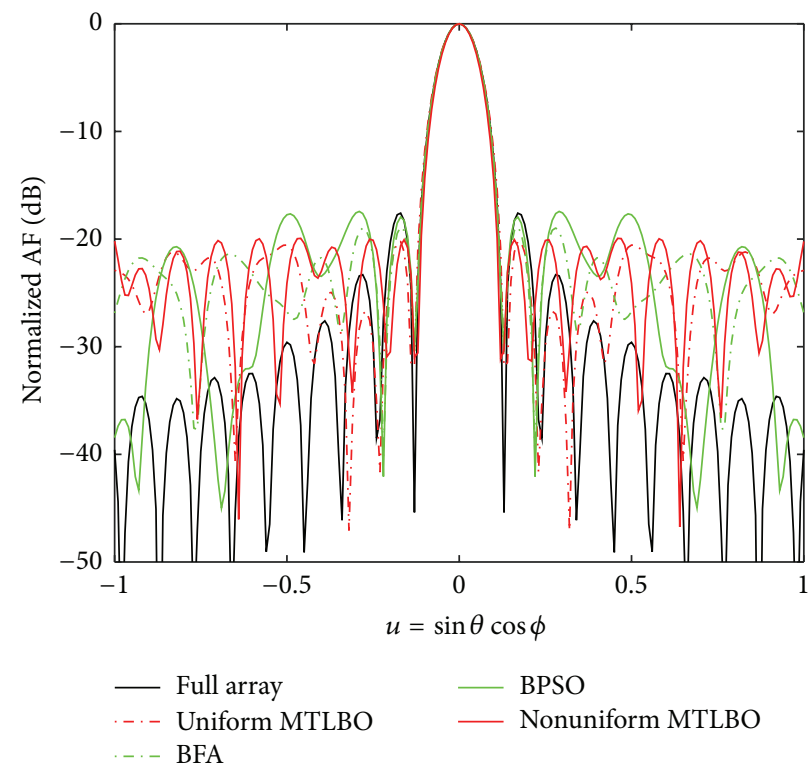

Figure 5: Comparison of array factors in the phi $=0^{\circ}$ plane with $\mathrm{FNBW}=0.13$.

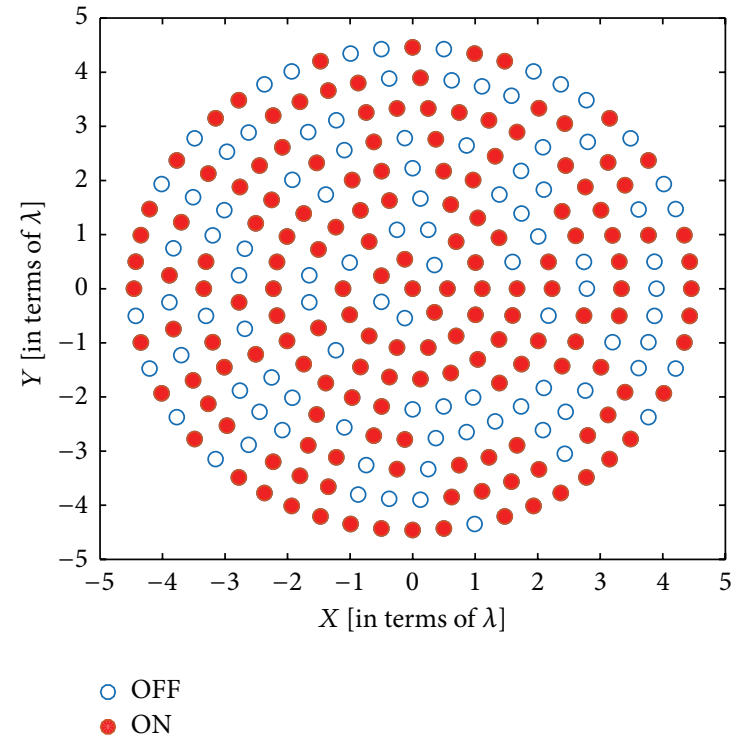

(a)

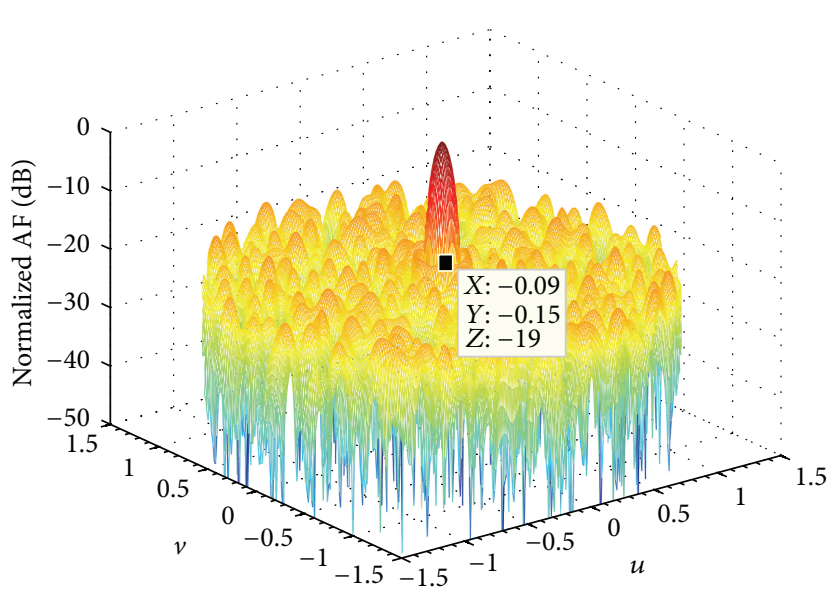

(b)

FIGURE 6: Uniform CCAA optimized by MTLBO for SLL reduction in basically extended space. (a) Configuration of a 156-element uniform array and (b) the related array factor with $\mathrm{SLL}=-19.0 \mathrm{~dB}$ and $\mathrm{FNBW}=0.13$.

$u-v$ space as the last simulation case. In this case, the $u-v$ space is extended to $[-2,2]$, which includes all combination of the arrival and steering directions. A uniform thinned CCAA was optimized by MTLBO for SLL reduction in the adequately extended $u-v$ space. The configuration of the 194-element array and the related array factor with a maximum SLL of $-16.03 \mathrm{~dB}$ are shown in Figure 8. The nonuniform CCAA was also optimized by MTLBO to get better array factor. As can be seen from Figure 9, the optimized nonuniform CCAA has $189 \mathrm{ON}$ elements, which saves 3 elements compared to the uniform CCAA; the SLL is also well controlled under
$-18.13 \mathrm{~dB}$ in the adequately extended $u-v$ space. The results of optimized arrays in both basically and adequately extended $u-v$ spaces are given in Table 2 . It is clearly shown that nonuniform CCAA can achieve better performance than uniform CCAA.

\section{Conclusions}

This paper describes the application of an optimization methodology termed MTLBO for synthesis of thinned concentric circular antenna arrays. The self-learning phase of 


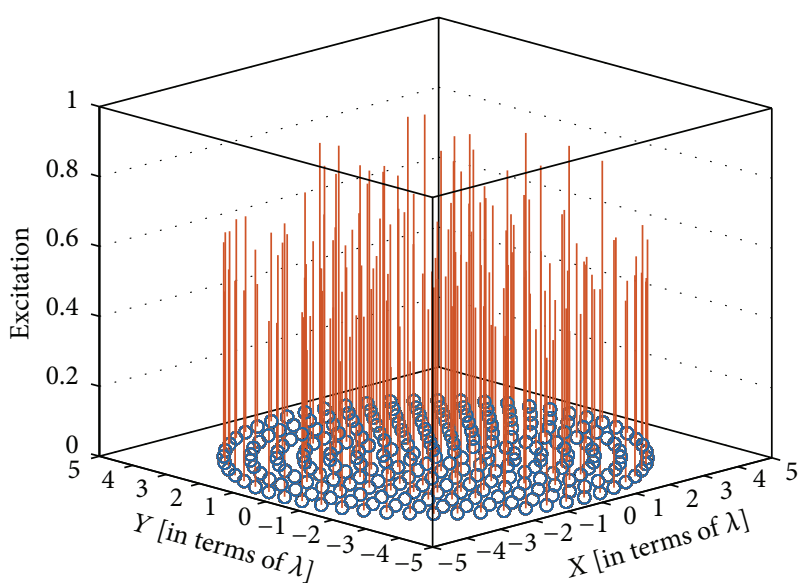

(a)

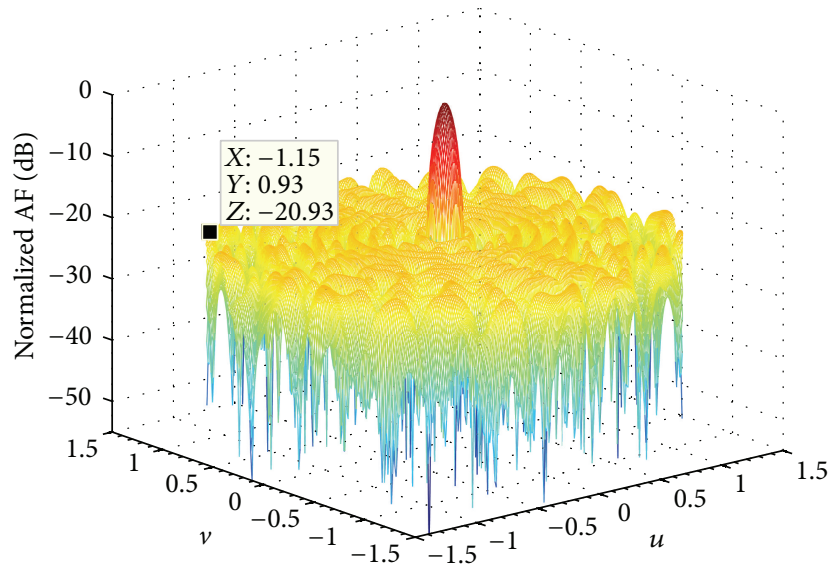

(b)

FIGURE 7: Nonuniform CCAA optimized by MTLBO for SLL reduction in basically extended space. (a) Configuration and excitation of a 152-element nonuniform array and (b) the related array factor with SLL $=-20.93 \mathrm{~dB}$ and FNBW $=0.13$.

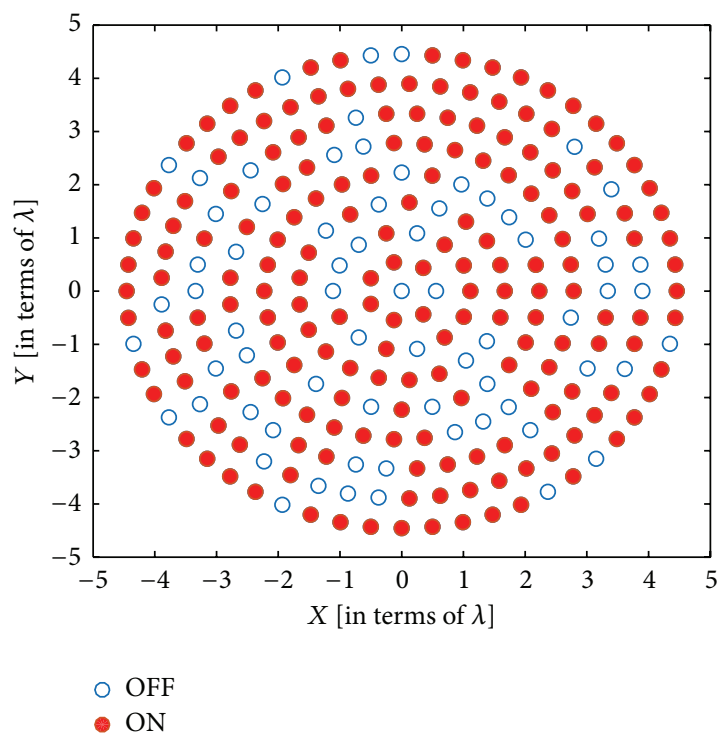

(a)

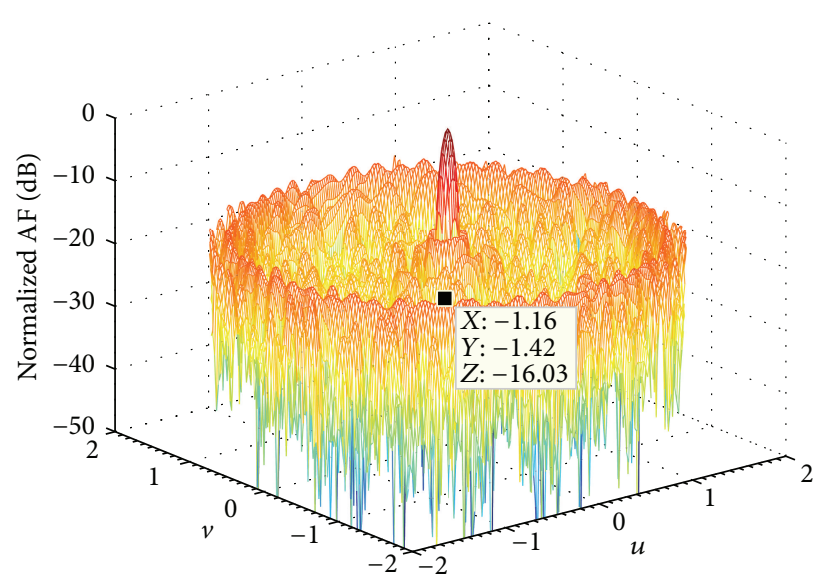

(b)

FIGURE 8: Uniform CCAA optimized by MTLBO for SLL reduction in adequately extended space. (a) Configuration of a 194-element uniform array and (b) the related array factor with $\mathrm{SLL}=-16.0 \mathrm{~dB}$ and FNBW $=0.13$.

TABLE 2: Results of thinned CCAA optimized by MTLBO for SLL reduction in the extended $u-v$ space.

\begin{tabular}{lcccc}
\hline Excitation & $u-v$ space & $\mathrm{Na}$ & SLL $(\mathrm{dB})$ & FNBW \\
\hline Uniform & {$[-1.5,1.5]$} & 156 & -19.00 & 0.13 \\
Nonuniform & {$[-1.5,1.5]$} & 152 & -20.93 & 0.13 \\
Uniform & {$[-2,2]$} & 194 & -16.03 & 0.13 \\
Nonuniform & {$[-2,2]$} & 189 & -18.13 & 0.13 \\
\hline
\end{tabular}

MTLBO is adjusted for CCAA design according to the geometry arrangement of antenna elements. The MTLBO algorithm is imposed to solve two important issues on CCAA: nonuniform CCAA design for better array factor and SLL reduction in the whole regular and extended space. A typical concentric circular antenna array has been synthesized with the objective of lowering SLL while keeping the $\mathrm{Na}$ and FNBW. Both uniform and nonuniform excitation amplitudes have been optimized for obtaining desired array factors in regular and extended space. Numerical results show that the proposed MTLBO outperforms other published works on SLL reduction in the regular $u-v$ space. The good performance of SLL reduction in the extended $u-v$ space shows that the MTLBO has potential applications in designing CCAAs for phased sonar and radar imaging systems. The MTLBO was demonstrated to be an effective method for 


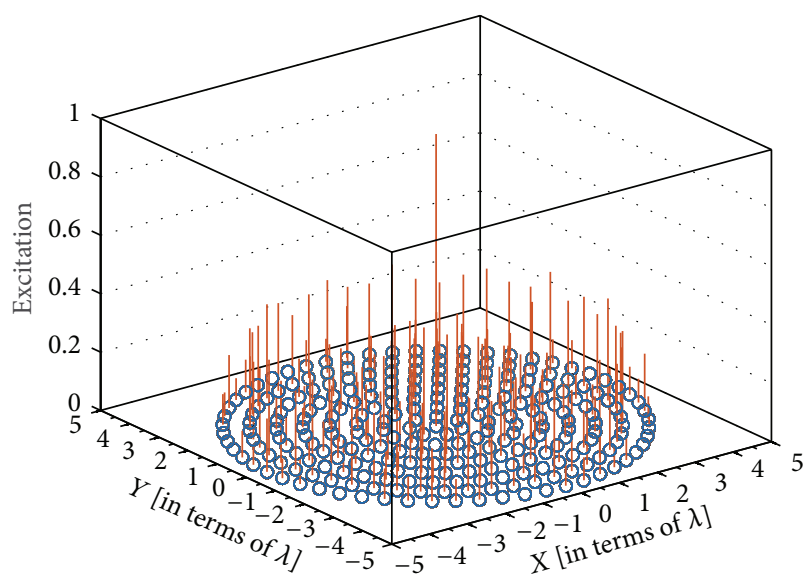

(a)

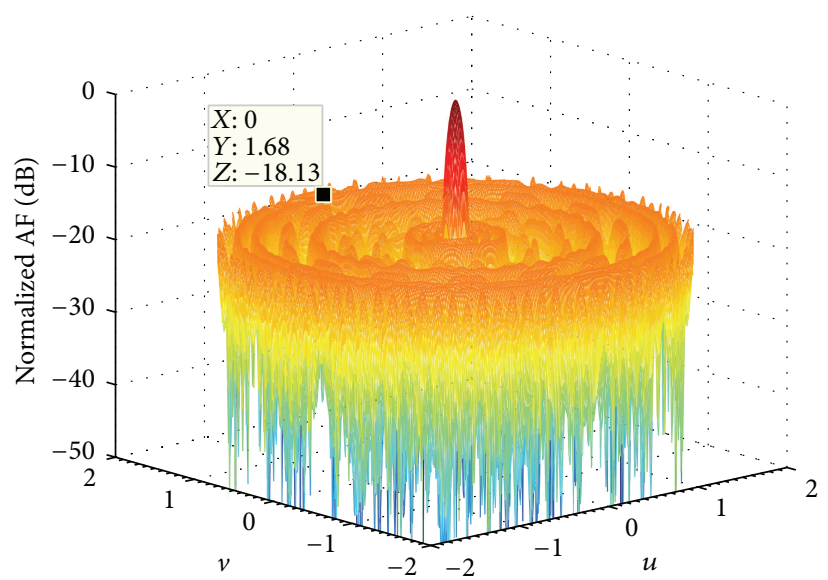

(b)

FIgURE 9: Nonuniform CCAA optimized by MTLBO for SLL reduction in adequately extended space. (a) Configuration of a 189-element uniform array and (b) the related array factor with $\mathrm{SLL}=-18.13 \mathrm{~dB}$ and FNBW $=0.13$.

CCAA optimization. It is expected to be a general method for optimization of other antenna arrays with different shapes and geometries.

\section{Conflict of Interests}

The authors declare that there is no conflict of interests regarding the publication of this paper.

\section{Acknowledgments}

This work was supported in part by the National Natural Science Foundation of China under Contract no. 51475177, no. 51121002, and no. 61175097, in part by the Doctoral Program of China under Contract no. 20110142130010, and in part by the Chinese Ministry of Public Security State Research Projects under Contract no. 2011ZDYJHJXY012.

\section{References}

[1] R. S. Elliot, Antenna Theory and Design, Wiley, Hoboken, NJ, USA, 2006.

[2] M. I. Dessouky, H. A. Sharshar, and Y. A. Albagory, "Optimum normalized-Gaussian tapering window for side lobe reduction in uniform concentric circular arrays," Progress in Electromagnetics Research, vol. 69, pp. 35-46, 2007.

[3] M. I. Dessouky, H. A. Sharshar, and Y. Albagory, "A novel tapered beamforming window for uniform concentric circular arrays," Journal of Electromagnetic Waves and Applications, vol. 20, no. 14, pp. 2077-2089, 2006.

[4] U. Singh and R. Munish, "Design of thinned concentric circular antenna arrays using firefly algorithm," IET Microwaves, Antennas \& Propagation, vol. 8, no. 12, pp. 894-900, 2014.

[5] M. I. Skolnik, J. W. Sherman III, and F. Ogg Jr., "Statistically designed density-tapered arrays," IEEE Transactions on Antennas and Propagation, vol. 12, no. 4, pp. 408-417, 1964.

[6] Y. H. Liu, Z. P. Nie, and Q. H. Liu, "Reducing the number of elements in a linear antenna array by the matrix pencil method,"
IEEE Transactions on Antennas and Propagation, vol. 56, no. 9, pp. 2955-2962, 2008.

[7] I. D. Dotlić and A. J. Zejak, "Arbitrary antenna array pattern synthesis using minimax algorithm," Electronics Letters, vol. 37, no. 4, pp. 206-208, 2001.

[8] T. Isernia, F. Ares, and O. M. Bucci, "A hybrid approach for the optimal synthesis of pencil beams through array antennas," IEEE Transactions on Antennas and Propagation, vol. 3, pp. 2301-2304, 2004.

[9] A. Trucco, "Thinning and weighting of large planar arrays by simulated annealing," IEEE Transactions on Ultrasonics, Ferroelectrics, and Frequency Control, vol. 46, no. 2, pp. 347-355, 1999.

[10] A. Trucco, "Weighting and thinning wide-band arrays by simulated annealing," Ultrasonics, vol. 40, no. 1-8, pp. 485-489, 2002.

[11] A. Trucco, M. Palmese, and S. Repetto, "Devising an affordable sonar system for underwater 3-D vision," IEEE Transactions on Instrumentation and Measurement, vol. 57, no. 10, pp. 23482354, 2008.

[12] R. L. Haupt, “Thinned arrays using genetic algorithms," IEEE Transactions on Antennas and Propagation, vol. 42, no. 7, pp. 993-999, 1994.

[13] M. A. Panduro and C. A. Brizuela, "Evolutionary multiobjective design of non-uniform circular phased arrays," COMPEL, vol. 27, no. 2, pp. 551-566, 2008.

[14] J. Wang, B. Yang, S. H. Wu, and J. S. Chen, "A novel binary particle swarm optimization with feedback for synthesizing thinned planar arrays," Journal of Electromagnetic Waves and Applications, vol. 25, no. 14-15, pp. 1985-1998, 2011.

[15] B. Basu and G. K. Mahanti, "Thinning of concentric two-ring circular array antenna using fire fly algorithm," Scientia Iranica, vol. 19, no. 6, pp. 1802-1809, 2012.

[16] U. Singh and T. S. Kamal, "Synthesis of thinned planar concentric circular antenna arrays using biogeography-based optimisation," IET Microwaves, Antennas and Propagation, vol. 6, no. 7, pp. 822-829, 2012.

[17] N. Pathak, G. K. Mahanti, S. K. Singh, J. K. Mishra, and A. Chakraborty, "Synthesis of thinned planar circular array 
antennas using modified particle swarm optimization," Progress in Electromagnetics Research Letters, vol. 12, pp. 87-97, 2009.

[18] P. Ghosh and S. Das, "Synthesis of thinned planar concentric circular antenna arrays-a differential evolutionary approach," Progress in Electromagnetics Research B, no. 29, pp. 63-82, 2011.

[19] U. Singh and M. Rattan, "Design of thinned concentric circular antenna arrays using firefly algorithm," IET Microwaves, Antennas \& Propagation, vol. 8, no. 12, pp. 894-900, 2014.

[20] A. Chatterjee, G. K. Mahanti, and A. Mahanti, "Synthesis of thinned concentric ring array antenna in predefined phi-planes using binary firefly and binary particle swarm optimization algorithm," International Journal of Numerical Modelling: Electronic Networks, Devices and Fields, vol. 28, no. 2, pp. 164-174, 2015.

[21] R. V. Rao, V. J. Savsani, and D. P. Vakharia, “Teaching-learningbased optimization: a novel method for constrained mechanical design optimization problems," Computer-Aided Design, vol. 43, no. 3, pp. 303-315, 2011.

[22] M. Črepinšek, S.-H. Liu, and L. Mernik, "A note on teachinglearning-based optimization algorithm," Information Sciences, vol. 212, pp. 79-93, 2012.

[23] N. Dib and A. Sharaqa, "Synthesis of thinned concentric circular antenna arrays using teaching-learning-based optimization," International Journal of $R F$ and Microwave Computer-Aided Engineering, vol. 24, no. 4, pp. 443-450, 2014.

[24] X. Chen, Z. Luo, X. He, and L. Zhu, “Thinning and weighting of planar arrays by modified teaching-learning-based optimization algorithm," Journal of Electromagnetic Waves and Applications, vol. 28, no. 15, pp. 1924-1934, 2014.

[25] R. L. Haupt, Antenna Arrays: A Computational Approach, John Wiley \& Sons, 2010.

[26] R. V. Rao and V. J. Patel, "An improved teaching-learning-based optimization algorithm for solving unconstrained optimization problems," Scientia Iranica, vol. 20, no. 3, pp. 710-720, 2013.

[27] R. V. Rao and V. Patel, "A multi-objective improved teachinglearning based optimization algorithm for unconstrained and constrained optimization problems," International Journal of Industrial Engineering Computations, vol. 5, no. 1, pp. 1-22, 2014.

[28] R. V. Rao and V. Patel, "Multi-objective optimization of two stage thermoelectric cooler using a modified teaching-learningbased optimization algorithm," Engineering Applications of Artificial Intelligence, vol. 26, no. 1, pp. 430-445, 2013. 

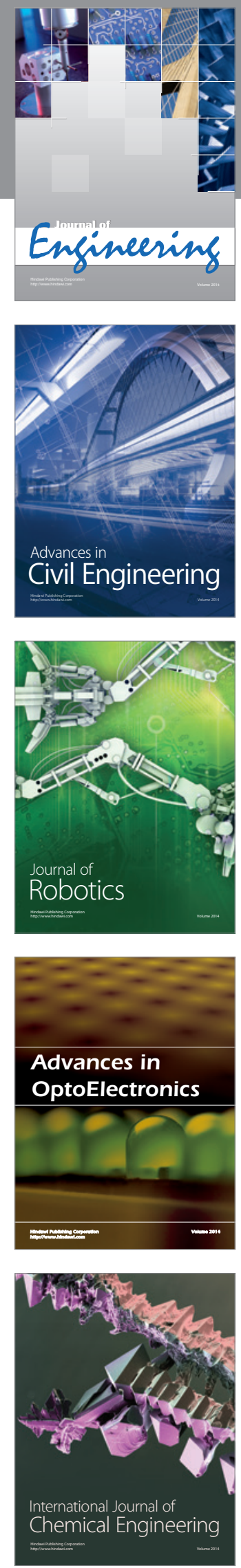

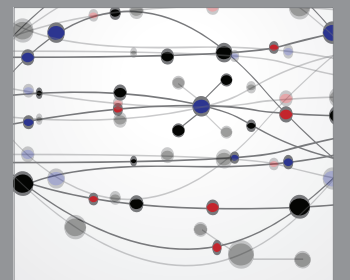

The Scientific World Journal
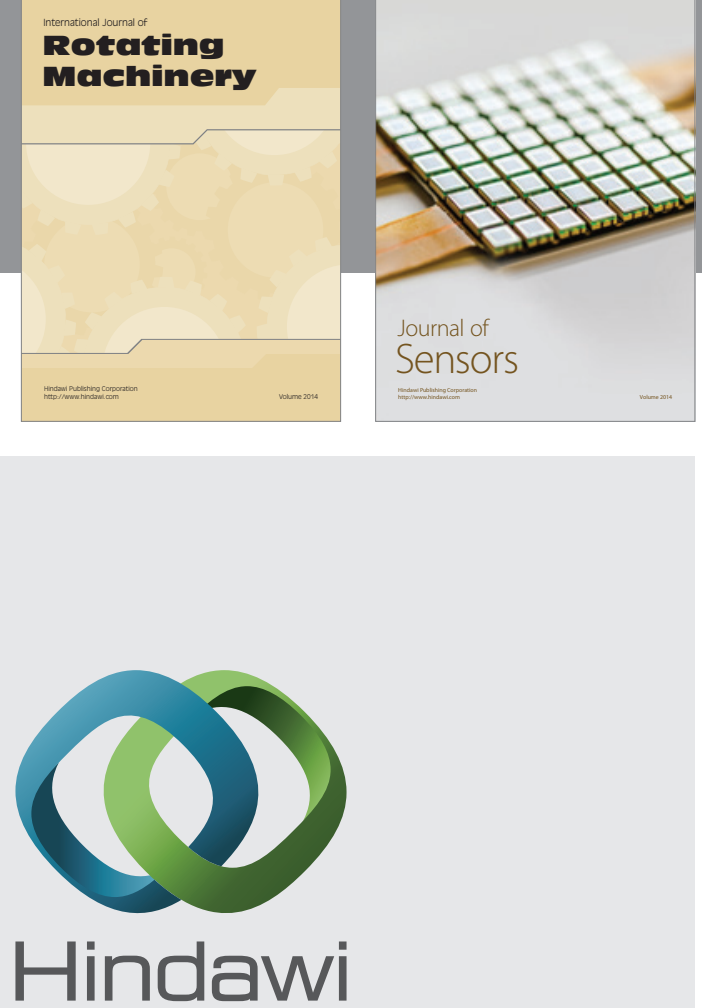

Submit your manuscripts at http://www.hindawi.com
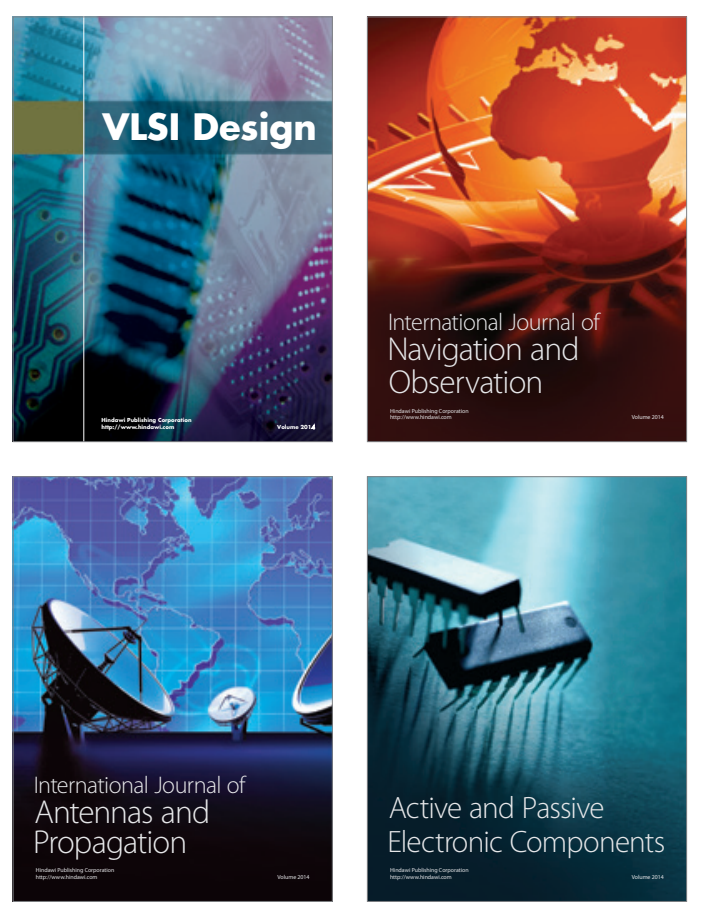
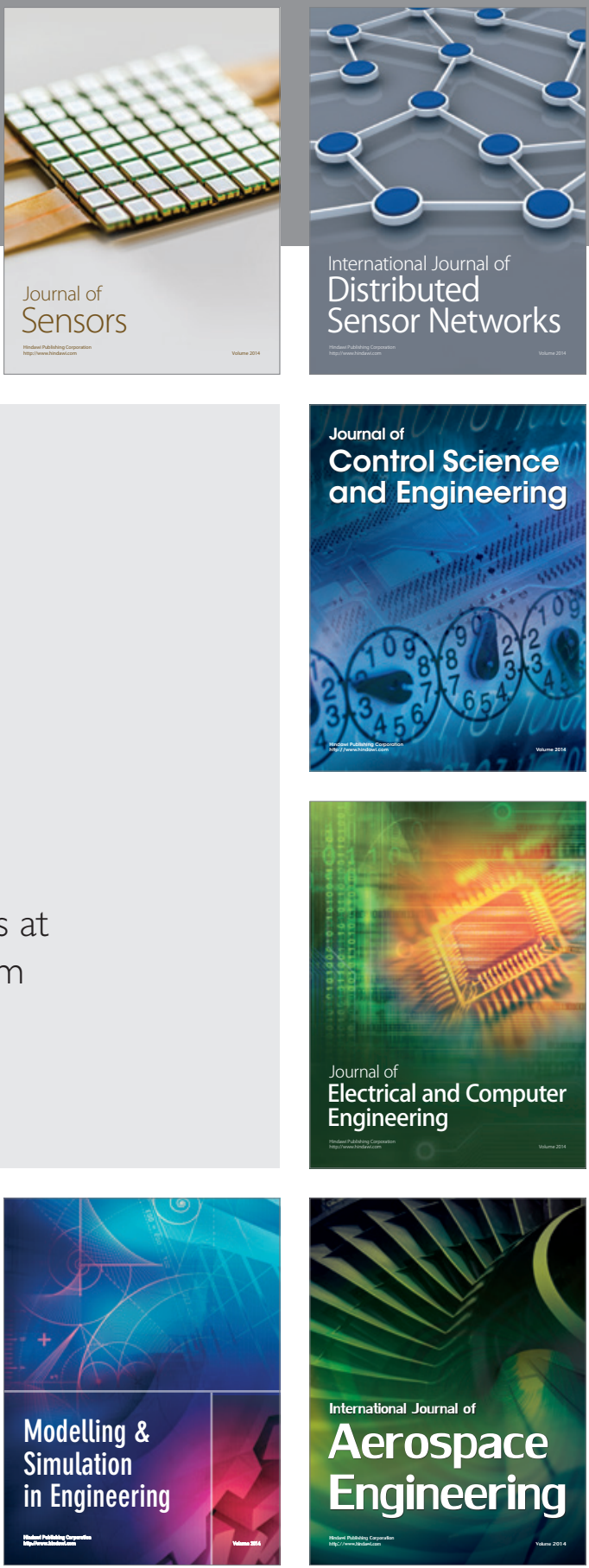

Journal of

Control Science

and Engineering
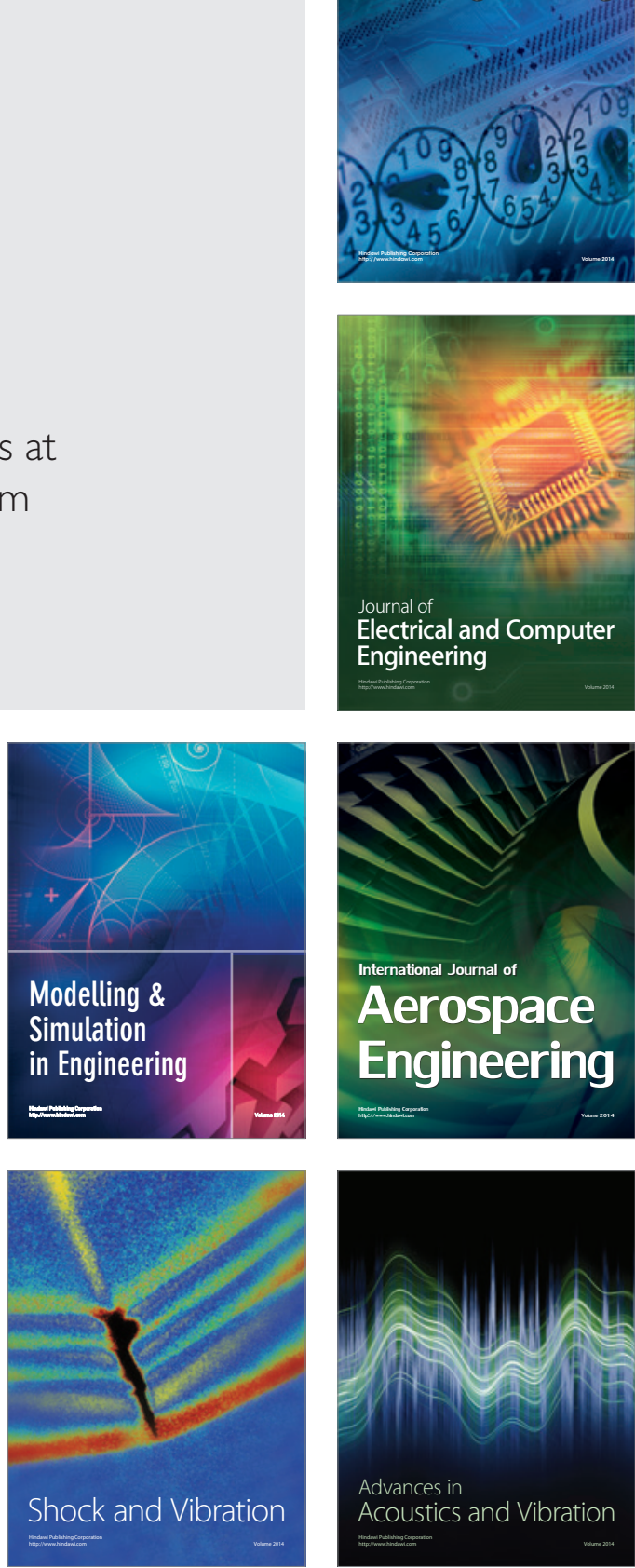